\title{
Static imaging of dynamic fluctuations in multiple light scattering media
}

\author{
Michael Heckmeier ${ }^{\mathrm{a}}$, Georg Maret ${ }^{\mathrm{b}}$ \\ ${ }^{a}$ Fakultät für Physik, Universität Konstanz, Postfach 5560, D-78434 Konstanz, Germany \\ ${ }^{\mathrm{b}}$ Institut Charles Sadron (CRM-EAHP), 6 rue Boussingault, F-67083 Strasbourg Cedex, France
}

\begin{abstract}
Multiple light scattering experiments in backscattering geometry are performed on highly viscous and solid turbid media which contain macroscopic inclusions of a low viscosity colloidal suspension. We demonstrate that the intensity probability distribution $P(I)$ of the multiple scattering speckle patterns can be used to image an inclusion even if its photon transport mean free path matches the value of the surrounding medium. The main difference between solid and liquid samples is discussed.
\end{abstract}

Dynamic speckle fluctuations of multiple scattered light have been widely investigated during the last years [1]. It has been shown that the normalized autocorrelation function of the multiple scattered electric field $g_{1}(t)$ provides accurate information about motion of the scattering particles in turbid suspensions. In recent work, the position-dependence of these dynamic fluctuations of multiple scattered light from macroscopically heterogeneous samples has been used to obtain images of objects which are hidden several photon transport mean free paths ${ }^{1} l^{*}$ inside a turbid medium [2-4].

On the other hand, the probability density of multiple light scattered speckles has been measured [5] and was shown to agree with the well-known situation of the scattered electric field in the single scattering case with a negative exponential distribution for the intensity.

In this paper we show how images of objects with internal dynamics can be generated from the determination of the speckle statistics. A new method is presented to visualize such a dynamic object buried in a turbid environment without explicitly probing the dynamics of the scat-

\footnotetext{
${ }^{1}$ The length over which the direction of propagation of diffusing light inside the sample is randomized.
}

tering particles inside the object. We demonstrate, that with simple static intensity measurements of the scattered light at different points of the sample's surface, it is possible to image an object even if its $l^{*}$ perfectly matches the value of its environment. The principle works as follows: Photons crossing the inclusion on their way through the multiple scattering medium pick up phase shifts due to the rapid brownian motion of scatterers inside the inclusion. We time-average the corresponding fast intensity fluctuations of the scattered light which gives a constant intensity value $\Delta I$. This intensity is treated as an incoherent background to the intensity statistics resolved on a slower time scale and resulting from the slower brownian motion of the scatterers of the environment medium. We show that this intensity shift $\Delta I$ is position dependent and can be used to create an image of dynamic heterogeneities. Thus, perhaps surprisingly, an object that does not provide any static scattering contrast can be visualized in a static light scattering experiment.

When coherent light is reflected from a rough surface or is scattered by a medium with random fluctuating refractive index, a random interference pattern is generated in the scattered light intensity [6]. This phenomenon, known as speckle, occurs in multiple light scattering when the coherence length of the used light source is larger than a 
typical photon path length inside the highly scattering medium. The statistical properties of speckle patterns have been widely investigated. Under the assumption of uncorrelated scatterers and uncorrelated scattering paths, the multiple scattered electric fields are independent random variables. According to the central limit theorem their sum (the total scattered electric field) has a Gaussian density function and hence, a negative exponential for the scattered intensity distribution $P(I)$ is obtained:

$P(I)=(1 /\langle I\rangle) \exp (-I /\langle I\rangle)$.

In a light scattering experiment the negative exponential density function is slightly modified: Because of the necessarily finite angular aperture of detection, light from more than exactly one speckle spot, or coherence area, hits the detector. This effect can be described approximately by a $\Gamma$-density distribution of the intensity [6]:

$P(I)=\frac{(\mu /\langle I\rangle)^{\mu} I^{\mu-1} \exp (-\mu I /\langle I\rangle)}{\Gamma(\mu)}$.

The parameter $\mu$ is proportional to the number of detected coherence areas and thus controls the crossover from the negative exponential distribution of Eq. (1) ( $\mu \rightarrow$ 1 , detector aperture goes to zero) to a narrow Gaussian density function centered around $\langle I\rangle$, corresponding to a completely averaged speckle pattern at very large aperture $(\mu \rightarrow \infty)$. For a $\Gamma$-density distribution the following relation is valid: $\left\langle I^{2}\right\rangle /\langle I\rangle^{2}=(\mu+1) / \mu$. Thus, $\mu$ can be extracted directly either by fitting a data set of $P(I)$ to Eq. (2), or by extrapolating the measured time autocorrelation function $g_{2}(t):=\langle I(0) I(t)\rangle /\langle I(0)\rangle^{2}$ to zero time.

In our experimental setup, a vertically polarized beam of a mono-mode $\mathrm{Ar}^{+}$-laser $(\lambda=514.5 \mathrm{~nm}$, beam waist $w \approx 1 \mathrm{~mm})$ is delivered on the light scattering cell as sketched in Fig. 1. To improve imaging of a buried object, the contribution of long scattering paths is increased by detecting only depolarized scattered light (V-H-scattering geometry). This excludes single scattering and reduces low order scattering paths since several scattering events are needed to change the polarization of the incoming light. A digital autocorrelator is used to determine the mean scattered intensity $\langle I\rangle$ and to measure the intensity correlation function $g_{2}(t)$ for a consistency check. Region (A) of our sample consists of monodisperse polystyrene beads (particle diameter $d=2.03 \mu \mathrm{m}$ ) suspended in a mixture of water $(60 \%)$ and glycerol $(40 \%)$ resulting in a photon transport mean free path of $400 \mu \mathrm{m}$. The buried capillary (B) is filled with a suspension of smaller PS-beads in pure water $(d=0.12 \mu \mathrm{m})$, matching the $l^{*}$-value of the environment (A) and therefore creates a dynamic heterogeneity [4]. The photon transport mean free paths were checked independently by dynamic multiple light scattering experiments in transmission geometry [1].

Fig. 2 shows typical autocorrelation functions which are measured in the described backscattering geometry. If

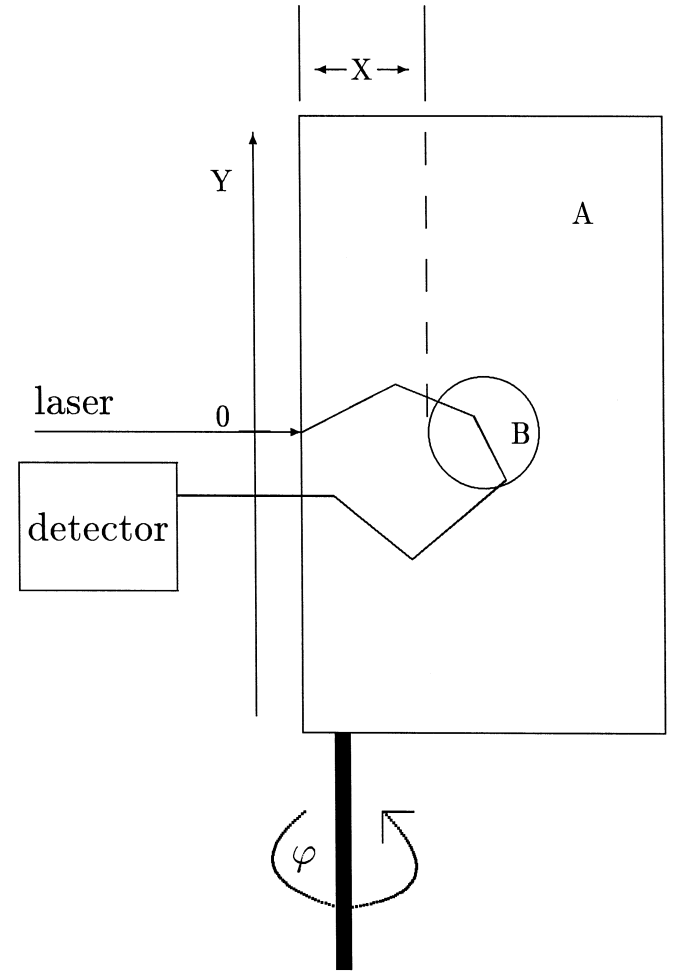

Fig. 1. Side view of the experimental setup. A large cell $(5 \mathrm{~cm} \times$ $5 \mathrm{~cm} \times 2 \mathrm{~cm}$ ) is filled with a colloidal suspension (A). (For the case of a liquid inclusion inside a stationary medium, this cell is replaced by a solid block of Teflon.) A cylindrical cavity (B) of diameter $1.5 \mathrm{~mm}$ and $10 \mu \mathrm{m}$ wall thickness contains a different suspension and can be placed at arbitrary positions inside the cell ( $y=0$ denotes centered laser entrance with respect to the capillary and for $x=0$, the wall of the cylinder is in contact with the inner frontside of the cell). The scattering cell can be rotated by about $\pm 5^{\circ}$ with respect to the detector axis.

the capillary is positioned inside the cell, there are two contributions to the decay of $g_{2}(t)$. The faster one $(t \approx 5$ $\left.\times 10^{-5} \mathrm{~s}\right)$ corresponds to the brownian motion of the small scatterers inside the capillary. The second decay ( $t \approx 10^{-2} \mathrm{~s}$ ) is due to the slower brownian motion of the scatterers in the higher viscous mixture of glycerol and water (region (A)). We emphasize that the value $g_{2}(0)$ of the measured autocorrelation functions serve as an independent check of the $\mu$-parameter of the speckle distribution function (Eq. (2)) but are not exploited in this work to image the buried capillary.

The intensity distribution $P(I)$ is determined with a time resolution of $0.4096 \times 10^{-3} \mathrm{~s}$. The value of the time interval used for averaging is indicated in Fig. 2 and lies well between the two decays of the correlation functions. Hence, the fast speckle fluctuations due to the brownian motion of the small scatterers are completely averaged. However, on this time scale the speckle pattern due to the 


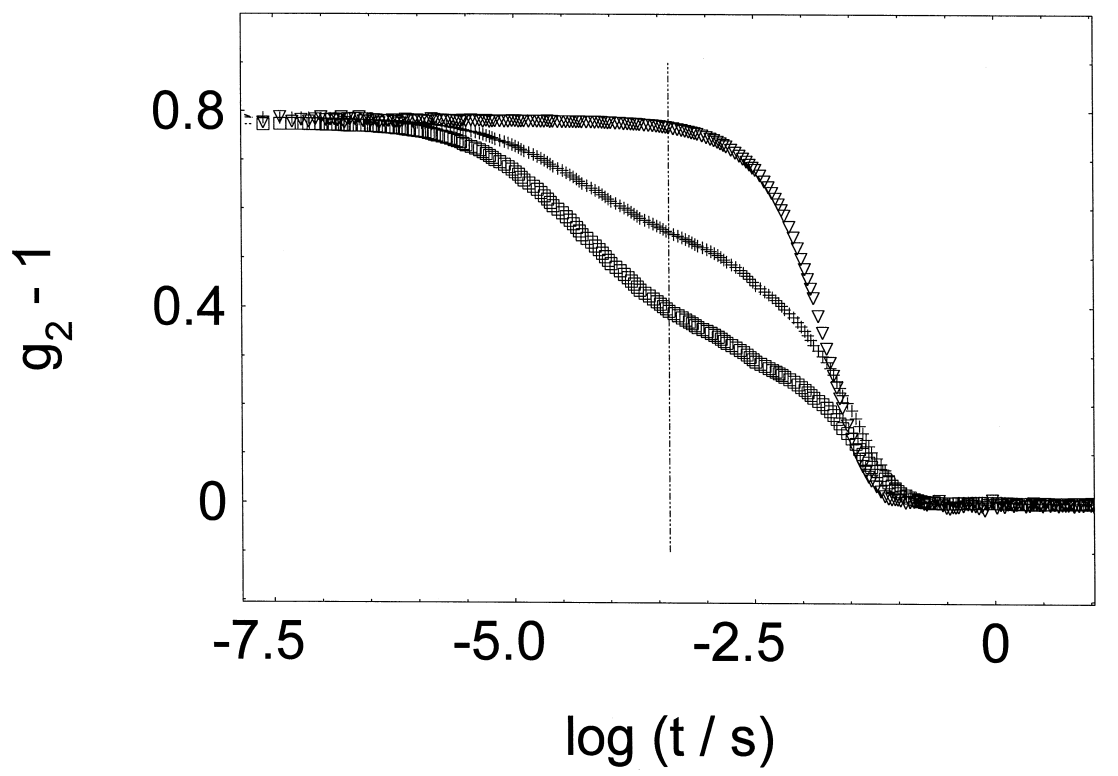

Fig. 2. Autocorrelation functions $g_{2}(t)$ for centered incidence of the laser beam with respect to the capillary's position $(y=0)$ and different depths of the capillary inside the liquid environment. $(\square) x=1.5 \mathrm{~mm} ;(+) x=2.5 \mathrm{~mm}$. $(\nabla)$ Homogeneous sample without included capillary. The vertical dashed line indicates the average time used for the static intensity measurements to determinate $P(I)$.

slower brownian motion of the scattering particles in region (A) is not yet averaged. Note, that this averaging is quite different from the angular preaveraging due to the finite detector size described by Eq. (2). The angular average leads to a finite probability for any intensity value $I>0$. However, the averaging due to the fast brownian motion of the scatterers theoretically results in a zero probability for small intensities ${ }^{2}$ up to a value $\Delta I$ which is directly related to the fraction of backscattered photons that crossed region (B) on their way through the sample. We take this empirically into account by introducing $\Delta I$ as a shift-parameter in Eq. (2):

$$
\begin{aligned}
P(I)= & (\mu /\langle I\rangle)^{\mu}(I-\Delta I)^{\mu-1} \\
& \times \frac{\exp [-\mu(I-\Delta I) /\langle I\rangle]}{\Gamma(\mu)} .
\end{aligned}
$$

Typical experimental intensity distributions $P(I)$ are presented in Fig. 3. The measured intensities are normalized with respect to the mean scattered intensity $\langle I\rangle$. The observed difference between data and theoretical curves is typical for experimentally determined probability distributions $P(I)[5,6]$. This can be explained with the influence of geometric details (form of aperture, size of light spot) of the intensity detection. Thus, Eqs. (1) and (2) are only coarse approximations. The $\mu$-parameter was obtained in-

\footnotetext{
${ }^{2}$ As seen from Fig. 3, this zero probability is not completely obtained experimentally.
}

dependently by fitting the $P(I)$-data without included capillary with Eq. (2), resulting in $\mu=1.36$ which is in good agreement with the intercept of the autocorrelation functions in Fig. 2. This $\mu$-value was used as a fixed parameter for the description of the data with Eq. (3) and therefore the solid curves in Fig. 3 are one-parameter-fits, $\Delta I$ being the only adjustable parameter. The position dependence of the obtained values $\Delta I$ is evident from Fig. 3 . If the laser enters the sample centered with respect to the capillary, a higher fraction of photons scanned the region (B). Due to the fast brownian motion of the small beads, these photons pick up additional dynamic phase shifts. This results in a more blurred speckle pattern close to the capillary and therefore the probability density $P(I)$ becomes position dependent. This approach is confirmed by Fig. 4, where in addition to $\Delta I$ the points of inflection $I_{\text {inf }}$ of the increasing part of the $P(I)$-data are presented $(\triangle)$. (The points of inflection are normalized to coincide with $\Delta I$ for large $y$-positions.) As seen from Fig. 4 both $\Delta I$ and $I_{\text {inf }}$ change systematically with the capillary's position and therefore can be used to localize the heterogeneity. The qualitative agreement of the position dependence of $\Delta I$ and $I_{\text {inf }}$ confirms the approach of using the approximative Eqs. (2) and (3) to describe the $P(I)$-data. In our case, where the depth $x$ is comparable to the diameter of the hidden tube, one gets a blurred profile of the hidden cylinder and the widths of these curves correspond approximately to the diameter of the tube. The values of $\Delta I$ are about $5 \%$ of the average intensity, so the 


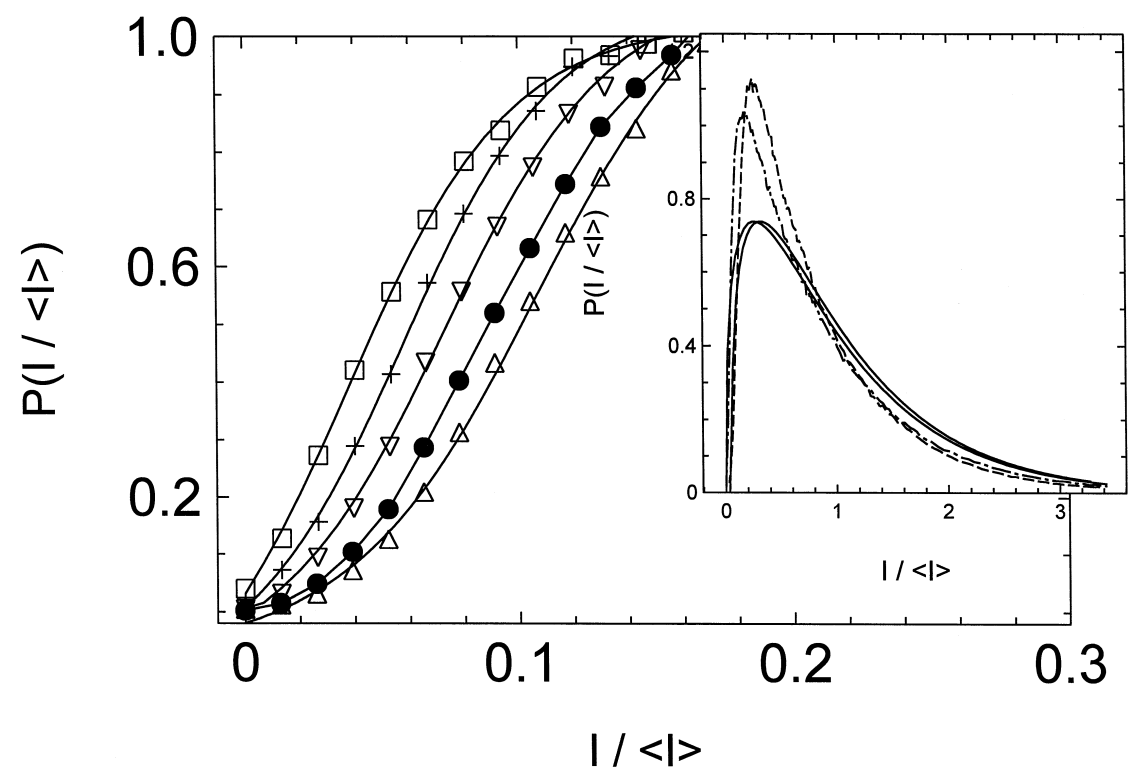

Fig. 3. Intensity probability distribution of the depolarized backscattered intensity for different capillary positions inside the higher viscous

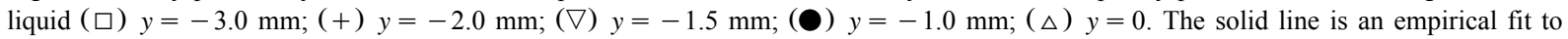
obtain the points of inflection of the $P(I)$-data for different positions of the capillary. The capillary is positioned at $x=1.5 \mathrm{~mm}$. Inset: Full range of the $P(I)$-data $(y=3.0 \mathrm{~mm}$ and $y=0)$, dashed lines are experimental and solid lines are theoretical curves (Eq. (3)).

relative effect has the same order of magnitude than the contrast-parameter $\Delta g$ of the dynamic autocorrelation function in our previous work [4].

We now extend the described speckle method of imaging to the case of a brownian inclusion inside a solid medium. Hence, in Fig. 1 region (A) is replaced by a solid block of Teflon $\left(l^{*}=280 \mu \mathrm{m}\right)$ containing a cylindrical cavity (B) with a diameter of $2 \mathrm{~mm}$ which is located at $x=1.5 \mathrm{~mm}$. The cylinder is filled with a monodisperse suspension of polystyrene beads (particle diameter: 1.14

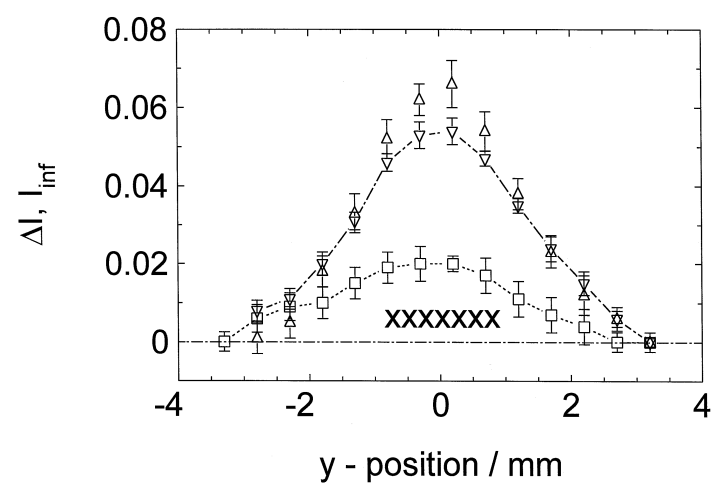

Fig. 4. Position dependence of the intensity parameter $\Delta I$ introduced in Eq. (3) for two different depths $x$ of the capillary. $(\nabla)$ $x=1.5 \mathrm{~mm}$; $(\square) x=2.5 \mathrm{~mm}$. $(\Delta) I_{\text {inf }}$ values of the points of inflection of the $P(I)$-data $(x=1.5 \mathrm{~mm}) . \times \times \times \times$ denotes the actual capillary position inside the surrounding colloidal suspension. $\mu \mathrm{m})$ with the same value of $l^{*}$ as in the solid environment. In this case, brownian motion of the scatterers occurs only in the confined region (B) and photons that did not scan this region create a stationary speckle pattern. The ensemble average is not performed "automatically" by the brownian motion of the scatterers in region (A) and the system becomes non-ergodic. To get the $P(I)$-data, different points of the stationary speckle pattern are detected by rotating the block by about $\varphi= \pm 5^{\circ}$ (cf. Fig. 1) with respect to the detector axis. The rotation time of $150 \mathrm{~s}$ results in a relaxation time in $g_{2}(t)$ of the order of $10^{-1} \mathrm{~s}$. Different values of the static intensity are measured again with a time resolution of $0.4096 \times 10^{-3} \mathrm{~s}$. In this way,

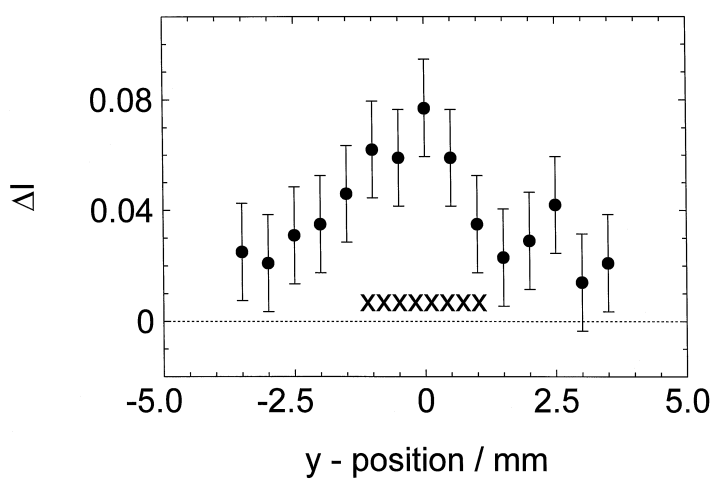

Fig. 5. $\Delta I$ as a function of the $y$-position of the cylinder inside the Teflon block. The cylinder is positioned at $x=1.5 \mathrm{~mm}$. The position of the inclusion is indicated by $\times \times \times \times$. 
ergodicity in the fluctuating light intensity is recovered. Using the same data analysis as above, we obtain the $\Delta I$-values shown in Fig. 5 . As in the case of the liquid environment, $\Delta I$ depends systematically on the position of the brownian inclusion. It therefore provides a means to image the buried cylindrical cavity. However, due to the restricted accuracy of the determination of the mean scattered intensity $\langle I\rangle$ by a solid sample, the error bars in Fig. 5 are larger than those in Fig. 4. The angular speckle size is approximately given by [6] $\lambda / w \approx 5 \times 10^{-4} \mathrm{rad}$. Hence, by rotating the sample by $\Delta \varphi=10^{\circ}=0.175 \mathrm{rad}$, about 340 speckle spots are averaged which results in an error of the mean intensity of $\pm 1 / \sqrt{340}$ which is about $5 \%$. This leads to the uncertainty in the measured intensity shifts $\Delta I$. Therefore, if the cylindrical inclusion is positioned deeper inside the Teflon block, the error bars are as large as the detected intensity shifts $\Delta I$ and the buried capillary can no longer be detected by analyzing the generated speckle patterns.

In conclusion, we have presented a new principle to image objects in multiple light scattering samples. This method based on measurements of the intensity distribution $P(I)$ and exploiting only static scattering intensities was applied to buried cavities inside turbid liquid and solid environments which do not provide static scattering contrast. Visualization of these cavities was possible by comparing with a slightly modified theoretical expression (3). The limits of this approach are presented. This simple method could be extended to obtain images in biological or medical applications. The speckle patterns could be detected by common equipment such as photo diodes or video cameras. However, our data evaluation using wellknown expressions for the intensity probability distribution $P(I)$ is only approximative. This calls for further theoretical work about speckle statistics from multiple scattering media, containing macroscopical inclusions.

\section{Acknowledgements}

M.H. expresses his gratitude for the financial support by the Deutscher Akademischer Austauschdienst (DAAD) through the HSPII/AUFE-program.

\section{References}

[1] D.Yu. Ivanov, A.F. Kostko, Opt. Spectrosc. 55 (1983) 950; G. Maret, P.E. Wolf, Z. Phys. B 65 (1987) 409; D.J. Pine, D.A. Weitz, P.M. Chaikin, E. Herbolzheimer, Phys. Rev. Lett. 60 (1988) 1134; D.A. Weitz, D.J. Pine, in: W. Brown (Ed.), Dynamic Light Scattering, Oxford Univ. Press, 1993, ch. 16, pp. 652-720; G. Maret, Current Opinion Colloid Interf. Sci. 2 (1997) 251.

[2] D.A. Boas, L.E. Campbell, A.G. Yodh, Phys. Rev. Lett. 75 (1995) 1855.

[3] M. Heckmeier, G. Maret, Europhys. Lett. 34 (1996) 257.

[4] M. Heckmeier, S.E. Skipetrov, G. Maret, R. Maynard, J. Opt. Soc. Am. 14 (1997) 185.

[5] P.E. Wolf, G. Maret, E. Akkermans, R. Maynard, J. Phys. France 49 (1988) 63.

[6] J.W. Goodman, in: J.C. Dainty (Ed.), Laser speckle and related phenomena, Springer, Berlin, 1984, ch. 2; J.W. Goodman, Statistical Optics, Wiley, 1985. 